\title{
CONTEXTUAL CONTROL OVER THE DERIVED TRANSFORMATION OF DISCRIMINATIVE AND SEXUAL AROUSAL FUNCTIONS
}

\author{
BRYAN ROCHE \\ National University of Ireland, Maynooth
}

\author{
DERMOT BARNES-HOLMES \\ National University of Ireland, Maynooth
}

\author{
YVONNE BARNES-HOLMES \\ National University of Ireland, Maynooth
}

\author{
PAUL M. SMEETS \\ University of Leiden, Holland
}

\author{
SIOBHÁN McGEADY \\ University of Bath, United Kingdom
}

A previous study by Roche and Barnes (1997) examined the transformation of conditioned sexual arousal in accordance with arbitrary relations. The current research replicated and extended that study by attempting to bring the derived transformation effect under contextual control. In Experiment 1, the functions of hand waving and clapping were first established for two nonsense syllables (called B1 and B2, respectively). Subjects were then exposed to relational pretraining, similar to that employed by Steele and Hayes (1991), in order to establish the contextual functions of Same and Opposite in two arbitrary stimuli. Subsequently, subjects were trained in the following relations; Same/A1-[B1-B2-N1], Same/A1-[C1-C2-N2], Opposite/A1-[B1-B2-N1], Opposite/A1-[C1-C2-N2] (underlined comparison stimuli indicate reinforced choices) from which the following relational responses emerged; Same/B1-C1; Same/B2-C2; Opposite/B1-C2; Opposite/B2-C1. During a testing phase, the stimulus functions established for $\mathrm{B} 1$ emerged for $\mathrm{C} 1$ in the presence of Same (i.e., the subjects waved) but those established for B2 emerged for $\mathrm{C} 1$ in the presence of Opposite (i.e., the subjects clapped). Similarly, the functions of B2 emerged for $\mathrm{C} 2$ in the presence of Same (i.e., the subjects clapped), but those established for B1 emerged for $\mathrm{C} 2$ in the presence of Opposite (i.e., the subjects waved). Experiment 2 established similar results using respondent eliciting functions in the place of hand clapping and waving.

The analysis of derived relational responding is both interesting and important because it has opened up new vistas of research into human language and complex behavior. Most research on derived relational responding has focused on the familiar stimulus equivalence effect.

This study was funded by the Research Seed Fund of the School of Social Sciences, University of Bath, England, where Bryan Roche was college lecturer from 1995 to 1997. The first author would like to thank Professor Helen Haste and the staff of the Department of Psychology, University of Bath for their kind support. He would also like to also thank Thomas S. Critchfield and an anonymous reviewer for several helpful suggestions. Address all correspondence concerning this article to Bryan Roche, Department of Psychology, National University of Ireland, Maynooth, Co. Kildare, Ireland. (Tel: +353 1 7086026; E-mail: BRYAN.T.ROCHE@May.ie). 
Studying this effect typically involves training subjects on a series of conditional discriminations (e.g., choose a stimulus, B1, given A1, and choose $\mathrm{C} 1$ given $\mathrm{A} 1$ ). Following this training, most subjects will choose $\mathrm{A} 1$ given $\mathrm{B} 1$ and $\mathrm{A} 1$ given $\mathrm{C} 1$ (i.e., symmetry), and choose $\mathrm{C} 1$ given $\mathrm{B} 1$ and $B 1$ given $C 1$ (i.e., transitivity) without reinforcement. When a subject demonstrates this performance the three stimuli, $A, B$, and $C$, are said to participate in an equivalence relation.

Although the equivalence paradigm has been employed in the analysis of a variety of complex human behaviors, such as social categorization (Watt, Keenan, Barnes, \& Cairns, 1991), sexual categorization (Roche \& Barnes, 1996), sexual stereotyping (Moxon, Keenan, \& Hine, 1993), and self-awareness (Dymond \& Barnes, 1995), it is nevertheless limited in empirical and conceptual scope. More specifically, the conceptualization of equivalence as a type of stimulus class permits only one such type of derived relation (see Hayes \& Barnes, 1997). Relational Frame Theory (RFT), however, extends the analysis of derived relational responding by treating equivalence as just one instance of this phenomenon (Barnes \& Roche, 1996; Dymond \& Barnes, 1995; Hayes \& Barnes, 1997; Hayes \& Hayes, 1989; Roche \& Barnes, 1996, 1997; Steele \& Hayes, 1991).

To date, several studies have provided empirical evidence that it is possible for human subjects to respond in accordance with relations other than equivalence, such as Opposition and Difference (Barnes \& Roche, 1996; Roche \& Barnes, 1996; Steele \& Hayes, 1991), and More Than and Less Than (Dymond \& Barnes, 1995). Stimulus relations such as Difference, Opposition, More Than, and Less Than are defined by different behavioral patterns. Whereas equivalence always yields the same derived relations across pairs of stimuli in a set (i.e., if $A$ is equivalent to $B$ and $B$ is equivalent to $C$, then $A$ and $C$ are also equivalent), the relations of Opposition and Difference do not. In the case of Opposition, if $A$ is the opposite of $B$ and $B$ is the opposite of $C$, then $A$ and $C$ are the same, not opposite. Similarly, if $A$ is different from $B$ and $B$ is different from $C$, then the relation between $A$ and $C$ is unspecified (i.e., $A$ and $C$ could either be the same, different, or opposite). Relational Frame Theory is important in the domain of language research, therefore, because it is the only account of relational activity that explains response patterns in accordance with relations other than equivalence (Barnes \& Roche, 1996; Hayes \& Barnes, 1997).

Relational Frame Theory is also important within the clinical domain because it places a special emphasis on the transfer of functions in accordance with networks of verbal stimuli. Indeed, this effect is a defining feature of derived relational responding from the RFT perspective (Hayes \& Hayes, 1989). A wide variety of stimulus functions have now been transferred in accordance with equivalence relations in the laboratory, including conditioned reinforcing functions (Hayes, Devany, Kohlenberg, Brownstein, \& Shelby, 1987), discriminative functions of public stimuli (Hayes et al., 1987), and private stimuli 
(DeGranpre, Bickel, \& Higgins, 1992), self-reports (Dymond \& Barnes, 1995), respondent eliciting functions (Dougher, Augustson, Markham, Greenway, \& Wulfert, 1994), and extinction functions (Dougher et al., 1994).

A simple and clinically relevant demonstration of the transfer effect was provided by Dougher et al. (1994). These researchers trained subjects on a series of interrelated conditional discrimination tasks that led to the emergence of two four-member equivalence relations (A1-B1C1-D1 and A2-B2-C2-D2). A mild electric shock applied to each subject's forearm then served as an unconditional stimulus (US) that followed presentations of B1 (i.e., respondent conditioning). Stimulus B2 was also presented, but in the absence of the US. Conditioned emotional responses to B1 and B2 were measured as skin conductance responses (SCRs). Subjects were then presented with some of the remaining members of each equivalence class to test for a transfer of eliciting functions. Of the 8 subjects, 5 showed evidence of respondent conditioning and a transfer of respondent eliciting functions. Dougher et al. suggested that these findings have important implications for our understanding of maladaptive and unexplained emotional behavior.

Although the Dougher et al. study provided some important insights into complex emotional behavior, the RFT account predicts even more complex forms of function transfer. In a previous study, Roche and Barnes (1997) exposed 6 subjects to relational pretraining designed to establish the contextual (relational) functions of Same and Opposite in two arbitrary stimuli. For instance, subjects were trained to relate same stimuli (e.g., a large line with a large line) in the presence of one contextual cue, and opposite stimuli (e.g., a large line with a small line) in the presence of a second contextual cue. Subsequently, subjects were trained on a series of conditional discriminations, with each discrimination being made in the presence of one of the two contextual cues. These tasks involved were Same/A1-[B1-B2], Same/A1-[C1-C2], Opposite/A1-[B1-B2], Opposite/A1[C1-C2], where all stimuli were nonsense syllables, and underlined comparisons indicate reinforced choices. During the relational testing phase subjects choose B1 given C1 and B2 given C2 in the presence of Same, whereas they choose $\mathrm{B} 1$ given $\mathrm{C} 2$ and $\mathrm{B} 2$ given $\mathrm{C} 1$ in the presence of Opposite. In summary, the emergence of the derived relations, [Same] B1-C1 and [Same] B2-C2, was observed during testing. Using a stimulus pairing procedure, sexual and nonsexual functions were then established for the two nonsense syllables, B2 and B1 (the respective functions of the stimuli were varied across subjects). Four of the five subjects who showed a significant emotional response differential to B1 and B2, as measured by skin resistance responses (SRRs), also showed a significant response derived differential between $\mathrm{C} 1$ and $\mathrm{C} 2$. These findings suggest that emotional responses can come under more complex forms of control than had hitherto been realized (see also Roche \& Barnes, 1997, 1998).

Interestingly, the functions established for B1 and B2 in Roche and Barnes (1997) do not appear to have transferred to C1 and C2, respectively, in the usual sense. More specifically, when sexual functions 
were established for B2 (for some subjects), these functions emerged for $\mathrm{C} 2$ by virtue of a trained opposition relation between $\mathrm{A} 2$ and $\mathrm{B} 2$ in combination with another trained opposition relation between $\mathrm{A} 2$ and $\mathrm{C} 2$ (i.e., both $\mathrm{C} 2$ and B2 were Opposite to A2 and so the Same as each other). In effect, the functions of B2 did not "transfer" directly to C2 but rather the functions of $\mathrm{C} 2$ were transformed in accordance with two combined opposition relations (i.e., C2 acquired sexual functions). According to RFT, this transformation of functions is a defining feature of derived relational responding, and equivalence is viewed as but one instance of the derived transformation effect. For this reason, the word "transformation," rather than "transfer," will be used throughout the present paper.

Before we outline the current research, it is important to highlight one important technical issue that arose in the Roche and Barnes (1997) study. Specifically, during the transformation of function test phase the $\mathrm{C} 1$ and $\mathrm{C} 2$ stimuli were presented in the absence of a contextual cue. In effect, it was assumed that under these conditions the eliciting functions established for the B stimuli would emerge for the C stimuli. Indeed, this assumption is consistent with RFT, which views Coordination (or equivalence) as the most common and fundamental type of verbal relation (see Barnes, 1994; Barnes \& Roche, 1996; Hayes \& Hayes, 1989). Nevertheless, although subjects may "default" to equivalence in the absence of an explicit contextual cue, it is not fair to say that such a performance emerges in the absence of contextual control. From a RFT perspective, relational responding is always under contextual control. Most children for instance have a long history of reinforcement for choosing the same stimulus in the context of matching games. In the absence of instructions to do otherwise, therefore, a matching-to-sample task functions as a contextual cue for pairing things that go together. Thus, the absence of an experimentally manipulated contextual cue in a matching context should itself function as the context for relating samples and comparisons according to a frame of Coordination (see also Fantino, 1998, for empirical evidence to this effect).

This view of relational activity maps directly on to real world language phenomena of interest to psychologists. For instance, in natural language a word presented on its own will elicit the functions of events that it "goes with," rather than those to which it is opposite or different (e.g., the word "hot" elicits functions of heat, not cold). Of course, words can also produce other functions when they are presented in the presence of contextual cues such as "what is the opposite of ... ?". Thus, RFT views relational responding as a thoroughly contextual affair, and the essence of this theory is to make empirical contact with the current and historical influences that give rise to relational responding. One implication of this theory, therefore, is that it should be possible to directly manipulate current context during a transformation of functions test similar to that used in Roche and Barnes (1997). Experiment 1 of the current study examined this possibility by attempting to contextually control the derived transformation of two discriminative stimulus functions for clapping and 
waving. Experiment 2 examined the possibility that the derived transformation of sexual eliciting functions would also come under further contextual control.

\section{Experiment 1}

\section{Method}

\section{Subjects}

Four male and three female unpaid volunteers between 18 and 27 years of age were recruited through notice board advertisements and personal contacts. All 7 subjects were undergraduate students from disciplines other than psychology.

\section{Apparatus}

Subjects were seated at a table in a small experimental room $(3 \times 3$ meters). The experimenter sat at the opposite side of the table facing the subject. All tasks were presented to subjects on laminated stimulus cards. All stimuli appearing on the cards were 36 point font for all stages of the experiment. Two stimuli, each consisting of a string of six characters (i.e., !!!!!! and $\% \% \% \% \% \%$ were used as contextual cues (i.e., Same and Opposite). For each subject these stimuli were randomly assigned to the roles of Same and Opposite. Fourteen nonsense syllables were employed for the tasks (i.e., JOM, CUG, BEH, YIM, ROG, DAX, PAF, VEK ZID, MEL, NEP, MAU, LER, JUR). These were randomly assigned as sample and comparison stimuli for each subject and are labeled, in the interests of clarity, using the alphanumerics; A1, B1, B2, C1, C2, N1, N2, N3, N4, X1, Y1, Y2, Y3, Y4.

\section{Operant Conditioning}

The purpose of this training phase was to establish the nonsense syllables B1 and B2 as discriminative cues for hand clapping and hand waving, respectively. Before the beginning of the training phase subjects were delivered brief oral instructions as follows:

During this stage of the experiment I will be showing you two laminated cards. Each card has an image printed in the center. I want you to look at that image and then I want you to either clap or wave your hands. Are you ready?

If the subjects indicated that they were not ready, the instructions were delivered again. The B1 and B2 stimuli were then presented to subjects in the center of a laminated card and in a quasi-random order (i.e., no more than two consecutive exposures to either stimulus). If a subject clapped following the presentation of the B1 stimulus, the experimenter said "Good, that is correct"; whereas if a subject waved when presented with this stimulus, the experimenter said "No, that is wrong." Conversely, 
if a subject waved following the presentation of the B2 stimulus, the experimenter said "Good, that is correct"; whereas if a subject clapped when presented with this stimulus, the experimenter said "No, that is wrong." This procedure continued until 10 consecutive correct responses to B1 and 10 consecutive correct responses to B2 were observed. All trials were followed by an intertrial interval of approximately $5 \mathrm{~s}$, during which time the experimenter recorded responses manually and retrieved the next stimulus card for presentation.

\section{Relational Pretraining, Training and Testing: General Sequence}

Relational pretraining, training, and testing tasks (see below) were presented manually by the experimenter on laminated stimulus cards. On all tasks the contextual stimulus (see below) appeared in the center, top third of the card, the sample stimulus appeared in the middle of the card, and three comparison stimuli appeared in a row at the bottom of the card. The stimulus card was presented to the subject face up on the experimental table and remained until the subject made a response by pointing to one of the comparisons. No observing response to the sample was required. The positions of comparison stimuli (i.e., left, middle, or right) on the stimulus cards were counterbalanced across trials.

Feedback (i.e., the spoken words "correct" or "wrong") followed responses on all training trials, and it was followed in turn by an intertrial interval of approximately $5 \mathrm{~s}$, during which time the experimenter recorded responses manually and retrieved the next stimulus card for presentation. During relational testing tasks, all feedback was omitted; responses were simply followed by the regular intertrial interval. Subjects were not informed that feedback would be terminated during this phase.

Same/Opposite pretraining and testing. The relational pretraining was designed to establish functions of Same and Opposite for the contextual cues (i.e., !!!!!!, \%\%\%\%\%\%) that would be used subsequently in the relational training and testing. Sample and comparison stimuli were related to each other along a physical dimension. For example, one set of comparison stimuli consisted of a long line, a medium line, and a short line. Thus, given a short-line sample stimulus, in the presence of the Opposite contextual cue, choosing the long-line comparison stimulus was reinforced. However, given the Same contextual cue and a short line, choosing the short-line comparison was reinforced. Four tasks constituted one problem set (i.e., Same/long line-long line, Same/ short line-short line, Opposite / long line-short line, Opposite / short line-long line). In total, there were eight problem sets, each consisting of four tasks.

The tasks for each problem set were presented in a quasi-random order in blocks of four trials, with each task presented once per block until the subject produced four consecutive correct responses. Subjects were then trained on a second problem set, and after four consecutive correct responses they were trained on a third problem set. Following four consecutive correct responses on this third problem set, tasks from all three problem sets were presented in a quasi-random order (i.e., one task 
from each problem set presented twice every six trials) until subjects produced six consecutive correct responses. Feedback was then terminated without warning, and subjects were tested on three novel problem sets $(4,5$, and 6). These were presented in a quasi-random order (one task from each of the three problem sets presented twice every six trials).

If subjects met the mastery criterion (i.e., produced six consecutive correct responses across the first six trials) the pretraining was terminated. If subjects failed to meet this criterion, they were retrained on problem sets 1 to 4 . Tasks were presented in a quasi-random order (i.e., one task from each of the 4 problem sets presented twice every 8 trials) until subjects produced eight consecutive correct responses. Feedback was then terminated and subjects were tested on problem sets 5,6 , and on a completely novel set 7 . These were presented in a quasi-random order (i.e., one task from each of the three problem sets presented twice every six trials), and if subjects met the mastery criterion the pretraining was terminated. If subjects failed to meet the criterion they were retrained on problem sets 1 to 5 . Tasks were presented in a quasi-random order (i.e., one task from each of the five problem sets presented twice every 10 trials) until subjects produced 10 consecutive correct responses. Feedback was then terminated and subjects were tested on problem sets 6,7 , and on a completely novel set 8 . These were presented in a quasi-random order (i.e., one task from each of the three problem sets presented twice every six trials), and if subjects met the mastery criterion the pretraining was terminated. None of the subjects failed at this level of testing.

Relational training. Immediately following relational pretraining subjects were exposed to the following training tasks; Same/A1-[B1-B2N1], Same/A1-[C1-C2-N2], Opposite/A1-[B1-B2-N1], Opposite/A1-[C1C2-N2], Same/X1-[Y1-B1-N3], Same/X1-[Y2-C1-N4], Opposite/X1-[Y3B2-N3], Opposite/X1-[Y4-C2-N4], where underlined comparison stimuli indicate reinforced choices. The $\mathrm{N} 1, \mathrm{~N} 2, \mathrm{~N} 3$, and $\mathrm{N} 4$ stimuli were included as incorrect comparison stimuli but were not employed during respondent conditioning or during the test for a transformation of function. These four latter training tasks were included to ensure that choosing $\mathrm{B} 1$ and $\mathrm{C} 1$ in the presence of Same, and choosing B2 and C2 in the presence of Opposite, would be reinforced on some trials, but not on others. This pattern of reinforcement was used to control for the possibility that the Same and Opposite cues could function as "mediating nodes" for simple equivalence relations between $\mathrm{B} 1$ and $\mathrm{C} 1$, and between $\mathrm{B} 2$ and $\mathrm{C} 2$, respectively (see Dymond \& Barnes, 1995, 1996, for detailed discussions of this issue).

Training occurred in blocks of 80 trials, with each of the eight tasks presented 10 times in a quasi-random order. Subjects were required to choose the correct comparison at least nine times across 10 exposures to each task to complete the relational training. The relational network can be seen in Figure 1. 

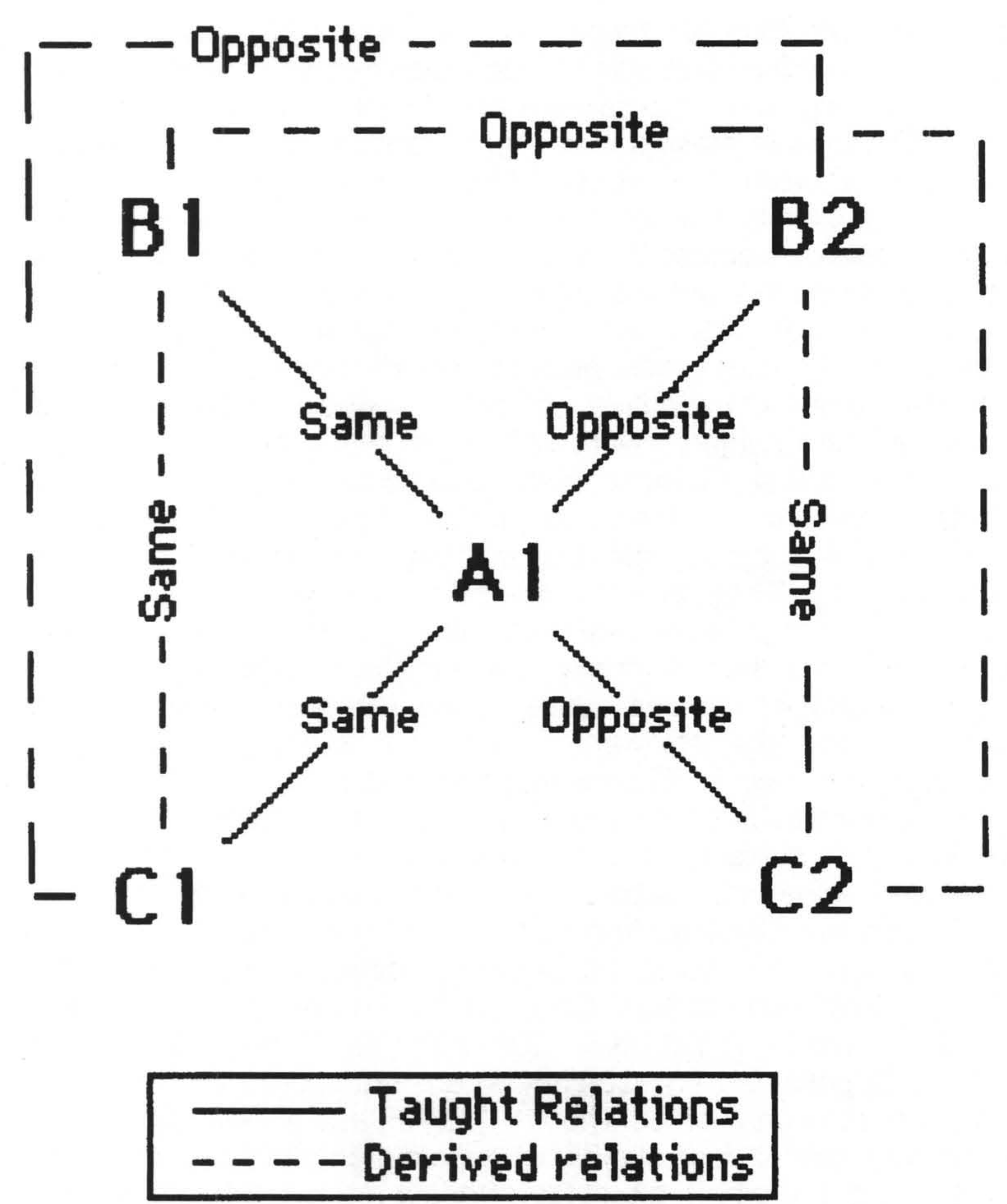

Figure 1. Diagrammatic representation of the relational network that was trained and tested in both Experiments 1 and 2.

Relational testing. The relational testing phase determined whether responding in accordance with the derived relations of sameness and opposition would emerge. The test tasks were as follows; Same/B1-[C1C2-N2]; Same/B2-[C1-C2-N2]; Opposite/B1-[C1-C2-N2]; Opposite/B2[C1-C2-N2]. Note that the $\mathrm{X}, \mathrm{Y}, \mathrm{N} 3$, and N4 stimuli were not presented to any of the subjects during relational testing. It was expected that subjects would (a) choose B1 given C1 in the presence of Same (i.e., B1 and C1 are both the same as $A 1$ and therefore the same as each other), (b) choose B2 given C2 in the presence of Same (i.e., B2 and C2 are both opposite to $A 1$ and therefore the same as each other), (c) choose B1 given $\mathrm{C} 2$ in the presence of Opposite (i.e., $\mathrm{B} 1$ is the same as $\mathrm{A} 1$, and $\mathrm{C} 2$ is opposite to $A 1$, and therefore $B 1$ is opposite to $C 2$ ), and (d) choose B2 
given $\mathrm{C} 1$ in the presence of Opposite (i.e., $\mathrm{B} 2$ is opposite to $\mathrm{A} 1$, and $\mathrm{C} 1$ is the same as $A 1$, and therefore $B 2$ is opposite to $C 1$ ). Testing occurred across blocks of 40 trials with each of the four tasks presented 10 times in a quasirandom order. If a subject failed to produce the predicted performance on 9 of 10 trials for each task, he or she was reexposed to the relational training and testing sequence up to a maximum of three times.

\section{Test for Transformation of Function}

No instructions were delivered to subjects at the outset of this phase, which began without warning immediately following the last trial of relational testing. During this phase, C1 and C2 were presented in the presence of both the Same and Opposite cues on laminated cards (i.e., the contextual cue assuming its regular position and the $C$ stimuli appearing in the center of the card). Thus, there were four stimulus cards used during this phase (i.e., Same/C1, Same/C2, Opposite/C1, Opposite/C2), presented in a quasirandom order (i.e., no more than two consecutive exposures to any stimulus) until all four cards had been presented 10 times each. All trials were followed by an intertrial interval of approximately $5 \mathrm{~s}$, during which time the experimenter recorded responses manually and retrieved the next stimulus card for presentation. It was agreed that a subject's participation would be terminated if a clapping or waving response was not observed within 2 minutes. All subjects responded within the time limit.

\section{Results and Discussion}

Two subjects failed to produce relation-consistent responding within four exposures to the relational training and testing sequence. These subjects were therefore dropped from the study and their performances are not discussed. The number of training trials required for the five remaining subjects on relational pretraining and relational training, respectively, were as follows: Matt: 32, 120; Christine: 54, 120; Simon: 51, 180; Ness: 37, 120; Gareth: 64,180 . Table 1 shows the performances of all 5 subjects on the relational testing phase of Experiment 1.

All subjects who produced a relation-consistent performance during the final relational testing phase subsequently demonstrated the

Table 1

Number of Relation-Consistent Responses for Each Testing Task in Experiment 1

\begin{tabular}{|c|c|c|c|c|c|c|c|c|c|c|c|}
\hline \multirow[b]{2}{*}{ Same-B1/C1 } & \multicolumn{2}{|c|}{ Matt } & \multicolumn{2}{|c|}{ Ness } & \multicolumn{2}{|c|}{ Simon } & \multicolumn{3}{|c|}{ Gareth } & \multicolumn{2}{|c|}{ Christine } \\
\hline & 10 & 10 & 10 & 10 & 310 & 10 & 2 & 21 & & 3 & 10 \\
\hline Same-B2/C2 & 10 & 10 & 10 & 10 & 910 & 10 & 2 & 2 & 9 & 7 & 9 \\
\hline Opposite-B1/C2 & 6 & 10 & 10 & 10 & 35 & 9 & 1 & 2 & 9 & 8 & 10 \\
\hline Opposite-B2/C1 & 6 & 10 & 7 & 10 & 27 & 9 & 1 & 4 & 9 & 3 & 10 \\
\hline
\end{tabular}

Note. Successive exposures to relational training and testing are represented from left to right in the data columns for each subject. Subjects were exposed to each test task 10 times during each exposure to the test phase. 
Table 2

Derived Hand Clapping Responses to Same/C1, Same/C2, Opposite/C1, and Opposite/C2 Probes During Transformation of Functions Test in Experiment 1

\begin{tabular}{lcccc}
\hline Subject & $\begin{array}{c}\text { Same } \\
\text { C1 }\end{array}$ & $\begin{array}{c}\text { Same } \\
\text { C2 }\end{array}$ & $\begin{array}{c}\text { Opposite } \\
\text { C1 }\end{array}$ & $\begin{array}{c}\text { Opposite } \\
\text { C2 }\end{array}$ \\
\hline Matt & 9 & 0 & 0 & 10 \\
Christine & 10 & 0 & 0 & 10 \\
Simon & 10 & 0 & 0 & 10 \\
Ness & 10 & 0 & 0 & 10 \\
Gareth & 10 & 0 & 0 & 9
\end{tabular}

Note. Subjects were exposed to each probe 10 times in a quasi-random order. Where subjects did not clap they waved (e.g., the number 9 indicates nine hand claps and one wave).

transformation of discriminative stimulus functions in accordance with the relational network. Specifically, following successful relational training and testing, the functions established for $\mathrm{C} 1$ emerged for $\mathrm{B} 1$ in the presence of Same (i.e., the subjects waved), but those established for B2 emerged for $\mathrm{C} 1$ in the presence of Opposite (i.e., the subjects clapped). Similarly, the functions of B2 emerged for $\mathrm{C} 2$ in the presence of Same (i.e., the subjects clapped), but those established for B1 emerged for $\mathrm{C} 2$ in the presence of Opposite (i.e., the subjects waved). Responses to the Same/C1, Same/C2, Opposite/C1, and Opposite/C2 probes are presented in Table 2.

In summary, these data demonstrate that the derived transformation of discriminative stimulus functions in accordance with complex relational networks is subject to even further forms of manipulable contextual control.

\section{Experiment 2}

Experiment 1 demonstrated that the derived transformation of discriminative functions is sensitive to contextual control, and that the psychological functions of stimuli can be determined by the history of relational responding applicable in current context. Although salient discriminative functions may readily transform in the manner demonstrated, however, it remains to be seen whether or not eliciting functions may also transform in this manner. Experiment 2 was designed to examine this issue. Experiment 2, however, is not a direct replication of Experiment 1. The procedures of both experiments differ because distinct and specialized procedures for analyzing the derived transformation of discriminative (see Dymond \& Barnes, 1995) and respondent eliciting functions (see Roche \& Barnes, 1995a, 1995b, 1997) have been developed by the first two authors.

\section{Method}

\section{Subjects}

Fifteen male volunteers between 18 and 26 years of age were recruited through notice board advertisements and personal contacts. 
Subjects were paid £5 Sterling (i.e., approximately \$7) for their participation, and another £5 Sterling if they completed relational training and testing successfully (see procedure section below). All subjects were undergraduate students from disciplines other than psychology.

\section{Apparatus}

Relational training and testing tasks were identical to those used in Experiment 1. However, during Experiment 2 all tasks were presented on an Apple Macintosh ${ }^{\circledR}$ computer (Model LCIII) that displayed black characters on a white background. Stimulus presentations and the recording of responses were controlled by the software application PsyScope (Cohen, MacWhinney, Flatt, \& Provost, 1993; see also Roche, Stewart, and Barnes-Holmes, 1999, for more specific information on this application and access to the current programs).

Differential eliciting functions were established for two nonsense syllables B1 and B2 using 45- to 60 -s film clips taken from a softpornography video production and a geographic documentary on the Scottish highlands. Sexual film clips depicted solo nude females simulating coitus and masturbation, whereas nonsexual clips depicted scenic landscapes. All film clips were played on a Ferguson ${ }^{\circledR}$ video player located in a monitoring room. Clips were relayed to subjects on a 20" Ferguson ${ }^{\circledR}$ television monitor located in the experimental room and placed directly beside the microcomputer used for relational training and testing (see procedure section below).

Respondent conditioning of general autonomic arousal was measured as Skin Resistance Responses (SRRs) on a Grass ${ }^{\circledR}$ polygraph (Model 7P1), which supplied a 10-microampere constant current through two $\left(1 \mathrm{~cm}^{2}\right)$ rim-sealed silver metal electrodes. The polygraph was located beside the video player in the monitoring room. Electrodes were prepared with an electrolyte that was produced from a Unibase (Parke Davis) and a $0.5 \% \mathrm{NaC} 1$ solution (Lykken \& Venables, 1971). Electrodes were secured to the electrode placement sites (see Procedure) with regular selfadhesive waterproof bandage (Ax, 1964).

At the end of the experiment a series of semantic differential scales were used to record subjects' ratings (from 1 to 5 ) of each of the probes: B1, B2, Same/C1, Same/C2, Opposite/C1, Opposite/C2, in terms of the degree to which each predicted the onset of a sexual film clip (where 5 indicated certainty that sexual material would follow and 1 indicated certainty that sexual material would not follow).

\section{Relational Pretraining, Training and Testing Procedure}

The tasks employed for the relational pretraining and testing phase were identical to those used in Experiment 1. However, in Experiment 2 two problem sets (each consisting of four tasks) were presented in a single block of pretraining. More specifically, each of the four tasks from problem set 1 were presented in quasi-random order until each had been presented twice (i.e., 8 trials). This procedure was then repeated for 
problem set 2 (i.e., 8 trials). Finally, the two sets ( 16 trials) were presented again in the same manner. In effect, a training block consisted of 32 training trials. Blocks were presented in succession until a subject's final 16 responses on a training block were correct (i.e., two exposures to each task across both problem sets). Testing involved the presentation of two novel problem sets, 3 and 4 . The four tasks from each problem set were presented only once in a quasi-random order (i.e., the four tasks from problem set 3 were presented in a random order, followed by the four tasks from problem set 4 , again presented in a random order). Subjects were required to produce correct responses on every one of the eight trials. If a subject failed to produce $100 \%$ correct responding they were retrained on problem sets 1 and 2 and on a novel set 5 (i.e., 48 trials in a block). When the subject produced correct responses on the final 16 trials of a training block they were retested on problem set 4 and on a novel set 6 . None of the subjects failed at this level of testing.

The relational training and testing procedure was identical to that used in Experiment 1 with the difference that all tasks were presented on a computer monitor. On all tasks (relational pretraining and testing, relational training and testing), the contextual stimulus (see below) appeared in the center, top third of the computer screen. The sample stimulus appeared $1 \mathrm{~s}$ later in the middle of the screen, and after a further 1-s delay, three comparison stimuli appeared in a row at the bottom of the screen. Contextual cue, sample, and comparison stimuli remained on the screen together, and no observing response to the sample was required. The screen position of the comparison stimuli (i.e., left, middle, or right) was counterbalanced across trials. Feedback (the printed words "CORRECT" or "WRONG") followed responses on all training trials, and was followed in turn by an intertrial interval of $2.5 \mathrm{~s}$, during which the screen remained blank. During relational testing tasks, all feedback was omitted; responses were simply followed by the intertrial interval. Subjects were not informed that feedback would be terminated during this phase.

\section{Respondent Conditioning}

Upon entering the experimental room each subject was required to read and sign a consent form (Appendix 1) acknowledging their awareness of the sexually explicit nature of some of the film clips that they were about to see. They were also informed that they were free to terminate their participation at any time and were asked not to discuss the study with anyone. Following the relational pretraining, training, and testing sequence (outlined above) subjects were read the following instructions.

During this phase of the experiment we are interested in examining electrical changes in your skin as you watch the film clips before you. The wires that I will attach to your index and middle finger will cause you no discomfort whatsoever. These wires do not allow us to "read your mind". All you are required to do now, is relax and watch the television monitor. If you have any questions please ask them now. 
Skin resistance electrodes were then prepared with electrolyte and applied to the volar surfaces of the distal phalanges of the index and middle finger of the left hand (Dawson, Schell, \& Filion, 1990). The experimenter then left to monitor SRRs from an adjacent room. Approximately $1 \mathrm{~min}$ after leaving the experimental room, the experimenter began relaying respondent conditioning trials to the subject's television monitor.

During conditioning trials one of the nonsense syllables, B1 and B2 $(7 \mathrm{~cm} \times 3 \mathrm{~cm})$ was presented in the center of the subject's monitor. This stimulus remained on the screen for $3 \mathrm{~s}$ and was followed by a 5 -s interval during which the monitor went dark. At the end of the 5-s interval a sexual or nonsexual US was presented following B1 and B2, respectively (i.e., a trace conditioning procedure). A simultaneous conditioning procedure was also employed on each conditioning trial, whereby the CS was flashed (once per second) periodically (every $15 \mathrm{~s}$ for $5 \mathrm{~s}$ ) in the top right corner of the television monitor during the presentation of the US. In effect, each respondent conditioning trial consisted of a combination of trace and simultaneous conditioning (see Chance, 1988, p. 52). Pilot work by the first two authors had previously indicated that this was a relatively reliable procedure for producing respondently conditioned SRRs (see Roche \& Barnes, 1995a).

The USs differed on every conditioning trial but were taken from the same pornographic video or nature documentary, respectively. The film clips varied from 45-60 $\mathrm{s}$ in duration. Intertrial intervals also varied from 45-60 s. Subjects were exposed to 12 trials (i.e., six exposures to both CSs), the order of which was randomized across subjects, with the restriction that neither CS could appear more than three times in succession.

In this procedure, the probability of a US presentation following a CS presentation was 0.8 (i.e., the US was omitted for one in five presentations of each CS). Omission trials were interspersed quasirandomly with conditioning trials (i.e., no more than four presentations of either CS were reinforced in succession). Thus, four or five presentations of each CS were followed by a US across each block of 12 trials. On those trials with no US, the CS was followed by the normal intertrial interval of 45-60 s, and the CS did not flash during the intertrial interval. Previous research by the first two authors (Roche \& Barnes, 1995a) had indicated that this procedure enhances resistance to extinction, thus facilitating repeated testing for the transformation of functions when USs are not presented. The preliminary respondent conditioning phase of the experiment lasted approximately $30 \mathrm{~min}$.

Subjects were exposed to a minimum of 24 trials (i.e., 12 exposures to each CS) in which B1 and B2 were followed on $80 \%$ of trials by sexual and nonsexual USs, respectively (i.e., no more than four successive presentations of either CS were followed by a US). Unbeknownst to the subject, recording of conditioned SRRs began on the seventh exposure to each CS (i.e., from the point of CS onset for the subsequent $5 \mathrm{~s}$ ). Responses to the CS were not contaminated by responses to the US 
because CS response measurement ceased $3 \mathrm{~s}$ before the onset of the US. The experimenter recorded a minimum of six responses to each CS, including omission trials. The experimenter terminated the training if visual inspection of the graphical representations of SRRs (continuously fed from the polygraph) suggested a response differential between B1 and B2 across the 12 respondent conditioning trials. The continuous graphical readout from the polygraph made it difficult to adhere to a strict criterion for judging an acceptable response differential, but, in general, training ceased when there appeared to be at least twice as many decreases in SRRs to B1 as to B2, or the majority of decreases in SRRs to $B 1$ were of greater magnitude than those to B2. When there did not appear to be a clear response differential, additional training trials were presented, with ongoing visual comparison of the effects of the two CSs. Training trials continued until (a) the experimenter judged that a clear response differential had emerged by applying the above criteria across all training trials, or (b) a maximum of 24 additional training trials had been presented (i.e., a maximum of 12 additional exposures to each stimulus, with US presentations on $80 \%$ of the trials). Where extra training trials were delivered, only the final six responses to both B1 and B2 were included in subsequent statistical analyses.

Three subjects failed to produce any discernible changes in skin resistance across 36 training trials, and thus these "stabiles" (see Augustson, Markham, \& Dougher, 1994) were dropped from the study, fully debriefed, and were paid $£ 5$ for their time (results are not presented).

\section{Test for the Transformation of Function}

The test for a transformation of eliciting functions in accordance with the relational network (see Figure 1) was administered to those subjects who demonstrated visual evidence of respondent conditioning. This test began immediately and without warning following the final respondent conditioning trial. That is, after the standard 45- to 60-s intertrial interval, one of the four probes, Same/C1, Same/C2, Opposite/C1, or Opposite/C2 was presented for $3 \mathrm{~s}$. All four probes were then presented in a quasirandom order (i.e., no more than two successive exposures to any probe) for $3 \mathrm{~s}$ per presentation until a subject had been exposed to each probe at least six times. During probe trials the contextual cue (i.e., Same or Opposite) was presented in the center top third of the screen $1 \mathrm{~s}$ before the $\mathrm{C} 1$ or $\mathrm{C} 2$ stimulus appeared beneath it in the center of the screen. An intertrial interval of $45-60$ s separated all probes. Skin resistance responses to probes were measured using the same procedures employed during respondent conditioning, from the point of stimulus onset for the subsequent $5 \mathrm{~s}$. The sexual and nonsexual USs were not relayed to the television monitor during this testing phase.

When each of the four probes had been presented six times, the test for a transformation of functions was complete. At this stage subjects were asked to respond to a series of semantic differential scales by rating, from 1 to 5 , the degree to which each of the probes; B1, B2, Same/C1, Same/C2, 
Opposite/C1, Opposite/C2 predicted the onset of a sexual film clip, where 5 indicated certainty that sexual material would follow and 1 indicated certainty that sexual material would not follow. This was done as a further measure of the transformation of stimulus functions.

\section{Response Quantification}

Phasic changes in skin resistance (SRRs) were used as the measure of respondent conditioning. In mathematical terms, skin resistance is simply the reciprocal of skin conductance (but see Roche \& Barnes, 1995b) and is measured in ohms $/ \mathrm{cm}^{2}$. Electrodermal activity (EDA) represents a relatively direct index of autonomic arousal (Dawson et al., 1990). Furthermore, as electrodermal responses are easily discriminable and quantifiable immediately following stimulus presentations, EDA represents a convenient measure of autonomic activity when experimental paradigms involve the repeated presentation of discrete stimuli.

Following extensive pilot testing (see Roche \& Barnes, 1995a, 1995b, 1997) an SRR was defined as the maximum absolute decrease in (ohmic) skin resistance, as compared with the skin resistance level taken at the time of stimulus onset, recorded within $5 \mathrm{~s}$ of stimulus onset. Increases in skin resistance (indicating relaxation) were not quantified but were read as having a value of zero and these zero values were included in all statistical analyses (see Roche \& Barnes, 1995b).

\section{Results and Discussion}

Three subjects failed to produce relation-consistent responding within four exposures to the relational training and were therefore dropped from the study. A further 3 subjects were dropped from the study because they failed to show clear visual evidence of respondent conditioning within 36 conditioning trials (see respondent conditioning procedure above). The performances of these 6 subjects will not be discussed. The number of training trials required for the 9 remaining subjects on relational pretraining

Table 3

Number of Relation-Consistent Responses on Each Testing Task in Experiment 2

\begin{tabular}{|c|c|c|c|c|c|c|c|c|}
\hline & Brendan & Chris & Damien & & & Ian & & Niel \\
\hline Same-B1/C1 & 10 & 10 & 10 & & 6 & 78 & 9 & 10 \\
\hline Same-B2/C2 & 10 & 10 & 10 & & 5 & 68 & 9 & 9 \\
\hline Opposite-B1/C2 & 10 & 10 & 10 & & 5 & 85 & 9 & 9 \\
\hline Opposite-B2/C1 & 9 & 10 & 10 & & 7 & 36 & 9 & 10 \\
\hline & Phil & Rik & \multicolumn{4}{|c|}{ Troy } & \multicolumn{2}{|r|}{ Roger } \\
\hline Same-B1/C1 & 10 & 1010 & 6 & 7 & 9 & & & 10 \\
\hline Same-B2/C2 & 10 & 810 & 5 & 4 & 9 & & & 10 \\
\hline Opposite-B1/C2 & 10 & 810 & 5 & 7 & 10 & & & 10 \\
\hline Opposite-B2/C1 & 10 & 1010 & 7 & 9 & 10 & & & 10 \\
\hline
\end{tabular}

Note. Successive exposures to relational training and testing are represented from left to right in the data columns. Subjects were exposed to each test task 10 times during any one exposure to the test phase. 
and relational training, respectively, were as follows: Brendan: 32,160 ; Chris: 128, 160; Damien: 32, 160; lan: 32, 160; Niel: 64, 320; Phil: 64, 160; Rik: 32, 160; Roger: 64, 160; Troy: 32, 240. Table 3 shows the performances of all 9 subjects on the relational testing phase of Experiment 2.

Given the known variability of electrodermal measures (Dawson, Schell, \& Filion, 1990; see also Roche \& Barnes, 1995a, 1995b) the most appropriate method of analysis for the current data is group means. Table 4 shows the average SRR (across subjects) to the conditioned stimuli, B1 and $\mathrm{B} 2$, and to each probe during the test for the transformation of functions. Table 4 also shows the standard deviation for each of these average responses, as well as the maximum and minimum responses observed during each trial type. A cross-subject statistical analysis indicated that respondent conditioning emerged at significant levels on a one-tailed $t$ test $(t=5.62 ; p=$ 0.002 ; see Figure 2). Transformations of this response differential were also observed at significant levels across subjects (see Figure 2).

Verbal reports recorded by the semantic differential scales were generally consistent with the physiological measures of response transformation (see Table 5). In summary, these data demonstrate that the derived transformation of respondently conditioned eliciting functions in accordance with relational networks can be brought under complex forms of contextual control, thereby extending the findings of Roche and Barnes (1997).

Table 4

Described Statistics for Cross-Subject Analysis of SRRs During Respondent Conditioning and Test for Transformation of Function in Experiment 2

\begin{tabular}{lllllll}
\hline & B1 & B2 & $\begin{array}{c}\text { Same } \\
\text { C1 }\end{array}$ & $\begin{array}{c}\text { Opposite } \\
\text { C1 }\end{array}$ & $\begin{array}{c}\text { Same } \\
\text { C2 }\end{array}$ & $\begin{array}{c}\text { Opposite } \\
\text { C2 }\end{array}$ \\
\hline Mean & 2.62 & 1.72 & 2.3 & 1.47 & 1.53 & 2.0 \\
Std.Dev. & 0.66 & 0.5 & 0.55 & 0.76 & 0.39 & 0.65 \\
Max. & 3.6 & 2.3 & 3.3 & 2.8 & 2.0 & 3.3 \\
Min. & 1.44 & 0.91 & 1.6 & 0.5 & 0.8 & 1.3 \\
\hline
\end{tabular}

Note. Note that raw SRRs were transformed according to the formula Log (SRR+1).

Table 5

Mean Ratings of Each Probe: B1, B2, Same/C1, Same/C2, Opposite/C1, and Opposite/C2, in Terms of Degree to Which Each Predicted Onset of a Sexual Film Clip

\begin{tabular}{cccccc}
\hline B1 & B2 & Same/C1 & Opp/C2 & Same/C2 & Opp/C1 \\
\hline 4.8 & 1 & 4.1 & 4 & 1.1 & 1.3 \\
\hline
\end{tabular}

Note. 5 indicated certainty that sexual material would follow and 1 indicated certainty that sexual material would not follow.

\section{General Discussion}

The findings of Experiments 1 and 2 appear to bear out the earlier suggestion that the transformation of both discriminative (Experiment 1) and respondent eliciting (Experiment 2) functions are contextually controlled 


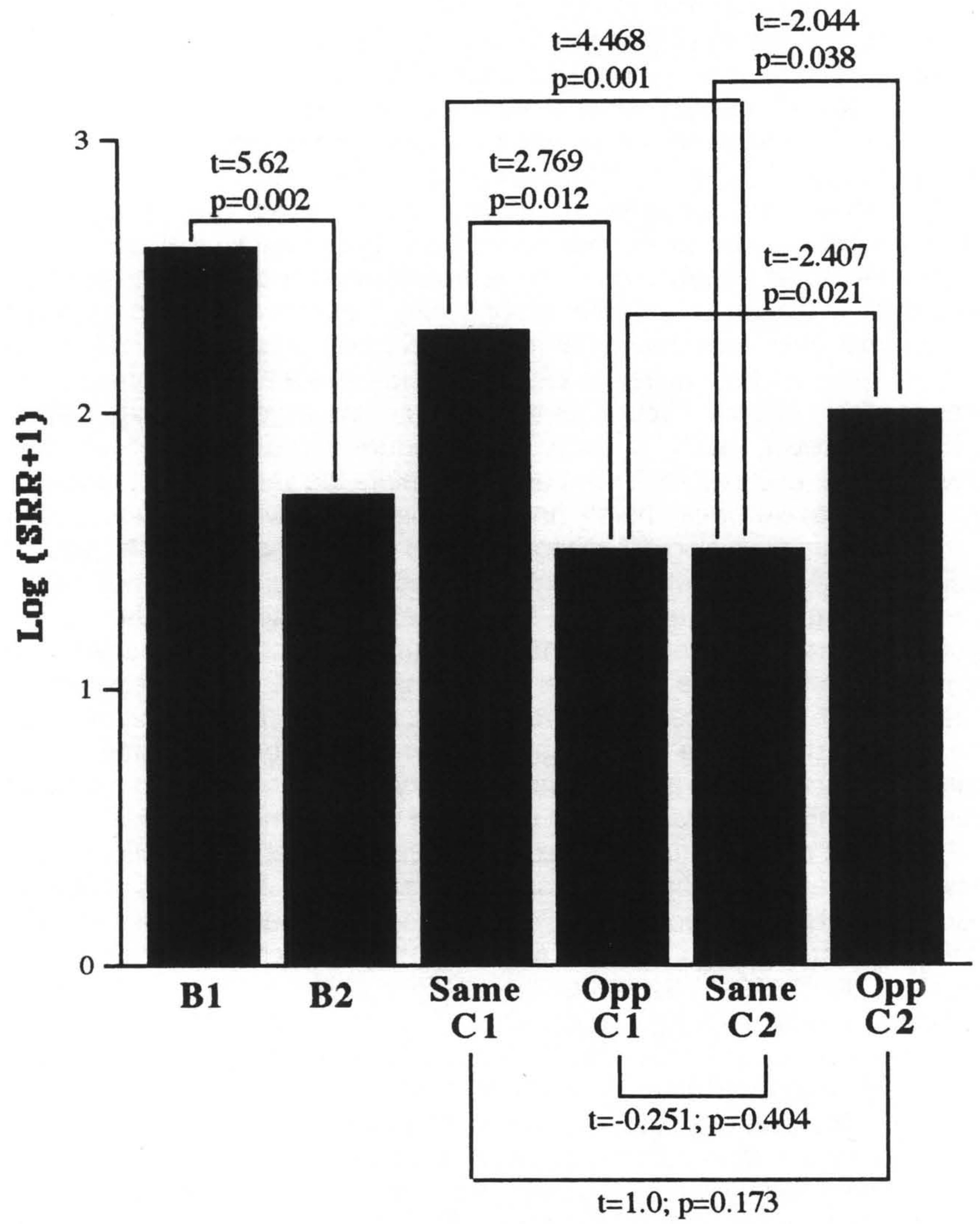

Figure 2. A group analysis of subjects' skin resistance responses during respondent conditioning (i.e., final six responses to both B1 and B2) and the transformation of functions test (i.e., Same/C1, Same/C2, Opposite/C1, and Opposite/C2) in Experiment 2. Black lines indicate the response differentials that were analyzed with a $t$ test for which $t$ and $p$ values are also provided.

events. These findings thereby support the idea that subjects in the Roche and Barnes (1997) study "defaulted" to equivalence in the absence of an explicit experimental contextual cue during the probes for a transformation of functions. The effects demonstrated in Experiments 1 and 2 of the current 
study also suggest that functions of both discriminative and eliciting stimuli (e.g., C1) may be determined not by mere participation in derived relations but (a) by the nature of those relations (i.e., Same and Opposite) and (b) contextual control over the transformation of functions.

The transformation of the verbal report measures obtained in Experiment 2 appear to parallel the transformation effects observed using the physiological measures. It is important to understand, however, that from a RFT perspective, these verbal reports did not necessarily mediate the physiological responses or visa versa. Both the physiological and verbal report measures simply recorded the transformation of different stimulus functions. Of course, the verbal reports may have recorded expectancies that did not arise until the verbal report sheet was presented. A talk-aloud procedure would be required to examine more closely the transformation of these verbal functions throughout the course of the experiment (see Hayes, 1986). However, even if such a procedure were to establish that physiological functions routinely transform before the verbal report functions (or visa versa) we would merely have identified a behavior-behavior relation (i.e., between physiological responses and verbal reports). This relation would not have complete explanatory status because it would not permit prediction and influence over the types of effects observed in the current study (see Hayes \& Brownstein, 1986). Future research would be required to determine the nature of the behavior-behavior relation between derived physiological and verbal report responses. At the present time we can conclude that both the physiological and verbal report measures in the current study emerged from the same history of conditioning and relational training and that they transformed according to the same relations.

With the current data the basic set of relational frame predictions have now been tested in the laboratory. Several studies have demonstrated derived relational responding in accordance with Same, Opposite, and Different relations (e.g., Dymond \& Barnes, 1995; Roche \& Barnes, 1996; Steele \& Hayes, 1991) as well as the relations of More and Less (Dymond \& Barnes, 1996; O'Hora, 1999). Other studies have demonstrated the transformation of both discriminative (Dymond \& Barnes, 1995, 1996) and eliciting functions (Roche \& Barnes, 1997) in accordance with such relations. One previous study has demonstrated contextual control over the transformation of discriminative stimulus functions (Wulfert \& Hayes, 1998), but the current study is the first to demonstrate such an effect in accordance with relations other than equivalence. Before we address some of the implications of these findings, we will consider some technical issues raised by the current data.

\section{Use of Arbitrary Contextual Cues}

To readers unfamiliar with the relational frame research literature, it may seem surprising that the words "Same" and "Opposite" were not used in place of the contextual cues in both Experiments 1 and 2. The principal reason for this is that the current study was in part a replication of Roche and Barnes (1997) in which abstract stimuli were used as contextual cues. Thus, 
it was important that the current research did not differ significantly from that earlier study. The words "Same" and "Opposite" may have functioned just as well, if not better, than the current contextual cues, because they have a longer history of use in the contextual control of verbal relations. Historically, however, real words have not been used in this type of research because one of the principal aims of the relational frame research agenda has been to make empirical contact with how contextual cues, both in the laboratory and in the natural environment, acquire their functional properties. Indeed, given the wealth of basic relational frame research that has now demonstrated control over derived relational responding, it may be superfluous to continue using abstract stimuli, rather than real words, as contextual cues in this type of research.

\section{Alternative Accounts of the Data}

Readers not favorable to the RFT account may attempt to explain the current effects in terms of a stimulus equivalence-based interpretation. Although several accounts of this type have been shown to be inadequate in many previous publications (Barnes, 1994; Barnes \& Roche, 1996; Dymond \& Barnes, 1995, 1996; Hayes \& Barnes, 1997; Roche \& Barnes, 1996; Steele \& Hayes, 1991), it may be worthwhile to consider an equivalence-based approach to the current relational frame data once again. Perhaps the most typical alternative view of the "transformation" effects observed in both Experiments 1 and 2 proceeds as follows. During relational training, there were two sample stimuli, $A 1$ and $X 1$. If subjects ignored these samples, and responded directly to the cues as sample stimuli, two equivalence classes may have formed in which the contextual cues participated as members (i.e., Same-B1-C1-Y1-Y2 [Class 1] and Opposite-B2-C2-Y3-Y4 [Class 2]). On this basis, we would expect that a response conditioned to $\mathrm{B} 1$ should transfer to $\mathrm{C} 1$ and a response conditioned to B2 should transfer to $\mathrm{C} 2$.

Now consider what actually happened during the test for the transformation of functions in Experiment 1. When $\mathrm{C} 1$ was presented with Same, subjects did indeed clap as the foregoing equivalence interpretation predicts (i.e., clapping was established for B1 and therefore transferred to Same and C1). When the Opposite cue was present with $\mathrm{C} 1$, however, subjects waved. This outcome is not clearly predicted by the equivalence-based interpretation because Opposite should control waving and $\mathrm{C} 1$ should control clapping. Similar problems arise for the remaining two probes: Same/C2 and Opposite/C2. On these probes subjects waved and clapped, respectively. The equivalence interpretation can not predict these consistent performances because during the Same/C2 probe the two stimuli controlled conflicting response topographies (i.e., clapping and waving, respectively), and during the Opposite/C2 probe both stimuli should have controlled waving, but did not. In effect, this account clearly predicts subjects' responses to only one of the four transformation probes.

One possible way to improve the predictive capacity of the 
equivalence-based interpretation is to assume control by the contextual cues only. Such control might take primacy over control by other class members (e.g., C stimuli) because, according to an equivalence-based interpretation, the contextual cues were related directly to the conditioned stimuli (B1 and B2) during relational training, but in contrast, the $\mathrm{C}$ stimuli were related to the conditioned stimuli indirectly through combined symmetry and transitivity. Perhaps therefore, the contextual cues served as more powerful controlling stimuli for clapping and waving than the $\mathrm{C}$ stimuli. Now, assuming the emergence of the two equivalence classes as before and their respective functions (i.e., Class 1: Same-B1-C1-Y1Y2/clap and Class 2: Opposite-B2-C2-Y3-Y4/wave), let us reconsider subjects' performances during the transformation test phase.

The second equivalence-based interpretation correctly predicts that given C1 with Same, subjects should clap. In addition, this account predicts, again correctly, that given $\mathrm{C} 1$ with Opposite, subjects should wave. Predictions for responses to the remaining two probes, however, are not consistent with the data. When C2 was presented with Same, subjects waved, whereas the equivalence account predicts that they should clap. In addition, when C2 was presented with Opposite, subjects clapped, whereas the equivalence account predicts that they should wave. In effect, the second equivalence-based interpretation makes correct predictions for only two of the four probes used during the transformation test.

A third equivalence-based account of the current data might involve the additional assumption that stimulus control shifted from contextual cues to the $\mathrm{C}$ stimuli across different transformation probes. Imagine, for example, that subjects attended to the contextual cue when $\mathrm{C} 1$ was present, but ignored it when $\mathrm{C} 2$ was present. Such an interpretation of the current data makes the additional correct prediction that subjects should wave when presented with Same and C2 (i.e., C2 is a Class 2 member and has acquired functions of clapping). Unfortunately for the current account, however, when C2 was presented with Opposite, none of the subjects waved. In effect, even this third equivalence-based interpretation predicts only three of the four probe outcomes. It is also difficult to identify the variables in the current study that could have produced this shift in stimulus control from the contextual cues to the $\mathrm{C}$ stimuli.

Furthermore, it is important to note at this point that an equivalencebased interpretation can not explain the performances observed during the relational training phases of both Experiments 1 and 2. More specifically, subjects clearly did not ignore the sample stimuli during relational training because clear control by sample stimuli was demonstrated during the training phase. For example, on one task responses to $\mathrm{B} 1$ were reinforced given $\mathrm{A} 1$ as a sample in the presence of Same (B2 was the incorrect comparison choice). However, on another task, responses to $\mathrm{Y} 1$ were reinforced when $\mathrm{X} 1$ was presented as a sample in the presence of Same, even though B1 was present as the incorrect comparison choice. Thus, any account of the transformation effects that relies on the idea 
that subjects ignored sample stimuli during training must contend with the fact that sample control was clearly shown in the relational training phases of both Experiments 1 and 2. Further evidence to challenge the suggestion that subjects ignore samples during relational training has been provided by Dymond and Barnes (1995). Still further publications have argued (Barnes \& Roche, 1996; Hayes \& Barnes, 1997) and demonstrated empirically (Dymond \& Barnes, 1995, 1996; Roche \& Barnes, 1996; Roche \& Barnes, 1997) that no other viable account of the current type of relational frame effect has yet been produced. In light of the foregoing, therefore, it would appear that an equivalence-based interpretation can not accommodate the current data satisfactorily.

\section{Wider Implications}

The current study extends the findings of Roche and Barnes (1997) by demonstrating that stimuli may acquire (sexual) functions through participation in derived relations but also by further contextual control over those relations. It is important to understand at this point, however, that the contextual control demonstrated here did not alter the relational network. More specifically, subjects' performances on the relational test demonstrated, for instance, that C1 was Opposite to C2. Although the Opposite/C2 and Same/C1 probes produced similar responses (i.e., sexual arousal in Experiment 2), C1 and C2 nevertheless remained opposite to each other. The contextual cues, however, modified the transformation of the stimulus $\mathrm{B}$ functions that emerged for the $\mathrm{C}$ stimuli. In effect, the relational network trained and tested in both Experiments 1 and 2 remained intact as the functions of stimuli were manipulated independently.

Interestingly, the independent manipulation of relational networks and the transformation of functions in accordance with those networks has recently become of interest to researchers in the field of Acceptance and Commitment Therapy (ACT, pronounced 'ACT' not A.C.T). A traditional approach to rehabilitative therapy for sexual offenders, for instance, has been to alter their thinking, or in behavioral terms, undermine relational networks. In the treatment of a rapist, for example, a therapist might use a cognitive relabeling strategy to undermine the offender's view of women as "needing to be controlled." One dramatic way in which this has been achieved in the past is by confronting rapists with rape victims in a nonsexual clinical setting (e.g., Murphy, 1990). From a behavioral point of view, this confrontation has the effect that the rape victim shifts from a frame of coordination perhaps containing "submission," or "deserving what they got" to a frame of coordination containing "a women like my mother or sister" or "a human being just like me."

The ACT approach does not focus on changing relational networks, however, but on the problematic transformation of functions in accordance with such networks. More specifically, ACT for sexual offenders might involve teaching clients to successfully "ride out" sexual urges without acting upon them. Even after a sexual offender has achieved this, they may still hold objectionable beliefs about women (i.e., 
some of the old verbal relations may still be intact). Nevertheless, transformations in accordance with these relations are now limited to mild sexual eliciting functions. The discriminative functions for sexual offending have been extinguished. In effect, the rapist may continue to experience urges to rape but he does not act them out (see LoPiccolo, 1994 for a detailed description of acceptance-based therapies in the treatment of sexual offenders).

The current study might represent a simple analog of the foregoing ACT outcome by bringing the transformation of B1 functions under the contextual control of the Opposite cue. For instance, during the relational test all subjects responded to C2 as Opposite to B1. Nevertheless, the test for transformation of functions presented conditions under which C2 produced the same response as B1 (i.e., when $\mathrm{C} 2$ was presented with Opposite). In effect, the relational network remained intact (i.e., B1 was Opposite to C2) while the functions of C2 were manipulated independently. The current study, therefore, represents one example of how RFT allows us to make empirical contact with highly complex behavioral effects of clinical relevance in a thoroughly functional analytic language. The challenge for future research will be to identify methods for establishing such clear contextual control over the transformation of functions within a clinical population.

\section{Conclusion}

The reader may argue at this point that the extrapolations arising from the current experimental analysis are premature. Indeed, considerable research will be needed in the future to examine more closely the relationship between the basic processes examined in the current study and sexual phenomena as they arise in the clinical setting. The fact that effects were observed in this highly contrived laboratory context, however, suggests that these basic processes may be at work, at least to some extent, in the world outside the laboratory.

If highly sophisticated research findings such as these are ever to have an application, basic researchers must attempt to build bridges from their own domain to that of the applied scientist. An important part of this endeavor will always be the construction of extrapolations. Of course, testing these extrapolations may stretch the limits of our scientific creativity, but if this is the challenge that presents itself, we should rise to it. If we are not mindful of our obligation to inform the research agenda of researchers in applied settings, we run the real risk of making our science self-sustaining and ultimately self-consuming. 


\section{References}

AUGUSTSON, E. M., MARKHAM, M. R., \& DOUGHER, M. J. (1994). Research in progress: A methodological note regarding human classical conditioning. Experimental Analysis of Human Behavior Bulletin, 12, 6-7.

AX, A. F. (1964). Goals and methods of psychophysiology. Psychophysiology, 1, 8-25.

BARNES, D. (1994). Stimulus equivalence and relational frame theory. The Psychological Record, 44, 91-124.

BARNES, D., \& ROCHE, B. (1996). Relational frame theory and stimulus equivalence are fundamentally different. The Psychological Record, 46, 489-507.

CHANCE, P. (1988). Learning and behavior (2nd ed.). Belmont, CA: Wadsworth.

COHEN, J. D., MACWHINNEY, B., FLATT, M., \& PROVOST, J. (1993). PsyScope: A new graphic interactive environment for designing psychology experiments. Behavioral Research Methods, Instruments \& Computers, 25(2), 257-271.

DAWSON, M. E., SCHELL, A. M., \& FILION, D. L. (1990). The electrodermal system. In J. Cacioppo \& L. G. Tassinory (Eds.), Principles of psychophysiology. Physical, social, and Inferential elements (pp. 295-324). New York: Cambridge.

DeGRANPRE, R. J., BICKEL, W. K., \& HIGGINS, S. T. (1992). Emergent equivalence relations between introceptive (drug) and extroceptive (visual) stimuli. Journal of the Experimental Analysis of Behavior, 58, 9-18.

DOUGHER, M. J., AUGUSTSON, E., MARKHAM, M. R., GREENWAY, D. E., \& WULFERT, E. (1994). The transfer of respondent eliciting and extinction functions through stimulus equivalence classes. Journal of the Experimental Analysis of Behavior, 62, 331-352.

DYMOND, S., \& BARNES, D. (1995). A transformation of self-discrimination response functions in accordance with the arbitrarily applicable relations of sameness, more than, and less than. Journal of the Experimental Analysis of Behavior, 64, 163-184.

DYMOND, S., \& BARNES, D. (1996). A transformation of self-discrimination response functions in accordance with the arbitrarily applicable relations of sameness, more than, and less than. Journal of the Experimental Analysis of Behavior, 46, 271-300.

FANTINO, E. (1998). Judgment and decision making: Behavioral approaches. The Behavior Analyst, 21, 203-218.

HAYES, S. C. (1986). The case of the silent dog - Verbal reports and the analysis of rules: A review of Ericsson and Simon's Protocol Analysis: Verbal reports as data. Journal of the Experimental Analysis of Behavior, 45, 351-363.

HAYES, S. C., \& BARNES, D. (1997). Analyzing derived stimulus relations requires more than the concept of stimulus class. Journal of the Experimental Analysis of Behavior, 68, 235-243

HAYES, S. C., \& BROWNSTEIN, A. J. (1986). Mentalism, behavior-behavior relations, and a behavior-analytic view of the purpose of science. The Behavior Analyst, 9, 175-190.

HAYES, S. C., DEVANY, J. M., KOHLENBERG, B. S., BROWNSTEIN, A. J., \& SHELBY, J. (1987). Stimulus equivalence and the symbolic control of behavior. Revista Mexicana de Analisis de la Conducta, 13, 361-374. 
HAYES, S. C., \& HAYES, L. J. (1989). The verbal action of the listener as the basis for rule-governance. In S. C. Hayes (Ed.), Rule-governed behavior: Cognition, contingencies, and instructional control (pp. 153-190). New York: Plenum Press.

LoPICCOLO, J. (1994). Acceptance and broad spectrum treatment of paraphilias. In S. C. Hayes, N. S. Jacobson, V. M. Follette, \& M. J. Dougher (Eds.), Acceptance and change: Content and context in psychotherapy (pp. 149170). Reno, NV: Context Press.

LYKKEN, D. T., \& VENABLES, P. H. (1971). Direct measurement of skin conductance. Psychophysiology, 8, 656-672.

MOXON, P. D., KEENAN, M., \& HINE, L. (1993). Gender-role stereotyping and stimulus equivalence. The Psychological Record, 43, 381-393.

MURPHY, W. D. (1990). Assessment and modification of cognitive distortions in sex. In W. L. Marshall, D. R. Laws, \& H. E. Barbaree (Eds.), Handbook of sexual assault (pp. 331-361). New York: Plenum Press.

O'HORA, D. (1999). The relational frames of Same, Opposite, More and Less: A response latency analysis. Thesis submitted for part-fulfillment of the requirements for a Bachelor of Arts Degree, Department of Applied Psychology, University College Cork, Ireland.

ROCHE, B., \& BARNES, D. (1995a). The establishment and electrodermal assessment of conditioned sexual responses. Experimental Analysis of Human Behavior Bulletin, 13, 26-29.

ROCHE, B., \& BARNES, D. (1995b). Measuring ill-defined events...and other problems. A reply to Augustson. Experimental Analysis of Human Behavior Bulletin, 13, 31-32.

ROCHE, B., \& BARNES, D. (1996). Arbitrarily applicable relational responding and sexual categorization: A critical test of the derived difference relation. The Psychological Record, 46, 451-475.

ROCHE, B., \& BARNES, D. (1997). A transformation of respondently conditioned stimulus function in accordance with arbitrarily applicable relations. Journal of the Experimental Analysis of Behavior, 67, 275-301.

ROCHE, B., \& BARNES, D. (1998). The experimental analysis of human sexual arousal: Some recent developments. The Behavior Analyst, 21, 37-52.

ROCHE, B., STEWART, I., \& BARNES-HOLMES, D. (1999). PsyScope: An easy-touse graphical system for designing and controlling equivalence experiments. The Experimental Analysis of Human Behavior Bulletin, 17, 5-7.

STEELE, D., \& HAYES, S. C. (1991). Stimulus equivalence and arbitrarily applicable relational responding. Journal of the Experimental Analysis of Behavior, 56, 519-555.

STEWART, I., \& ROCHE, B. (in press). PsyScope: An easy-to-use graphicoriented application for designing and controlling computer-based research on relational responding. The Experimental Analysis of Human Behavior Bulletin.

WATT, A., KEENAN, M., BARNES, D., \& CAIRNS, E. (1991). Social categorization and stimulus equivalence. The Psychological Record, 41, 33-50.

WULFERT, E., \& HAYES, S. C. (1988). Transfer of a conditional ordering response through conditional equivalence classes. Journal of the Experimental Analysis of Behavior, 50, 25-141. 


\section{Appendix 1}

I consent that I am willing to participate in this study. The purpose of the study has been satisfactorily explained to me and I expect to have any further information requested, regarding this study, supplied to me at the end of the experiment. I am aware that as a requirement of this study, I will be exposed to sexually explicit images of solo nude models. These models will not be engaged in any sexual acts and all images are of the type commonly available for purchase in high street stores. The total time spent viewing these images will last, approximately, from 15 to 40 minutes (the total time that all images will be actually on the screen will not exceed 15 minutes).

As a further requirement of the study I will have two fingers from my left hand attached to a set of electrodes used to record electrical activity in my skin. These electrodes will not cause any discomfort and I am free to remove them at any point should I so wish.

I am aware that the duration of and remuneration for the study depends upon my performance on a series of problem-solving tasks. The average time requirement is approximately 3.5 hours for the experiment as a whole. I will receive $£ 5$ remuneration for my participation and another $£ 5$ upon completion of the study. If I terminate the study, or fail to pass the problem solving phases I will be entitled only to $£ 5$ remuneration for participation, and not the $£ 5$ remuneration for completion.

I have not been coerced in any way to participate in this study and I understand that I may terminate my participation in this study at any stage if I so wish. I understand that my participation in this study has no bearing upon grades for academic work in the School of Social Sciences or the University of Bath, in general.

I am over 18 years of age.

Signed 
ROCHE ET AL. 